\title{
Comparison between preoperative and postoperative concurrent chemoradiotherapy for rectal cancer: an institutional analysis
}

\author{
Jeong Won Lee, MD¹, Jong Hoon Lee, MD¹, Jun-Gi Kim, MD², Seong Taek Oh, MD², \\ Hyuk Jun Chung ${ }^{2}$, Myung Ah Lee, MD³, Hoo Geun Chun, MD³, Song Mi Jeong, MD', \\ Sei Chul Yoon, MD¹, Hong Seok Jang, MD'1 \\ Departments of ${ }^{1}$ Radiation Oncology, ${ }^{2}$ Colorectal Surgery, and ${ }^{3}$ Medical Oncology, Seoul St. Mary's Hospital, \\ The Catholic University of Korea College of Medicine, Seoul, Korea
}

\begin{abstract}
Purpose: To evaluate the treatment outcomes of preoperative versus postoperative concurrent chemoradiotherapy (CRT) on locally advanced rectal cancer.

Materials and Methods: Medical data of 114 patients with locally advanced rectal cancer treated with CRT preoperatively (54 patients) or postoperatively (60 patients) from June 2003 to April 2011 was analyzed retrospectively. 5-Fluorouracil (5-FU) or a precursor of 5-FU-based concurrent CRT (median, $50.4 \mathrm{~Gy}$ ) and total mesorectal excision were conducted for all patients. The median follow-up duration was 43 months (range, 16 to 118 months). The primary end point was disease-free survival (DFS). The secondary end points were overall survival (OS), locoregional control, toxicity, and sphincter preservation rate.

Results: The 5-year DFS rate was 72.1\% and 48.6\% for the preoperative and postoperative CRT group, respectively ( $p=0.05$, the univariate analysis; $p=0.10$, the multivariate analysis). The 5 -year OS rate was not significantly different between the groups (76.2\% vs. $69.0 \%, p=0.23$ ). The 5 -year locoregional control rate was $85.2 \%$ and $84.7 \%$ for the preoperative and postoperative CRT groups $(p=0.98)$. The sphincter preservation rate of low-lying tumor showed significant difference between both groups $(58.1 \%$ vs. $25.0 \%$, $p=0.02)$. Pathologic tumor and nodal down-classification occurred after the preoperative CRT (53.7\% and 77.8\%, both $p<0.001)$. Acute and chronic toxicities were not significantly different between both groups ( $p=0.10$ and $p=0.62$, respectively).

Conclusion: The results confirm that preoperative CRT can be advantageous for improving down-classification rate and the sphincter preservation rate of low-lying tumor in rectal cancer.
\end{abstract}

Keywords: Rectal cancer, Concurrent chemoradiotherapy

\section{Introduction}

Colorectal cancer is the third most common cancer whose incidence is increasing in Korea [1]. Adjuvant chemoradiotherapy (CRT) is performed as the standard treatment following radical surgery in locally advanced rectal cancer to improve local control and overall survival (OS) $[2,3]$. There have been some attempts to search for more effective therapies [4]. In particular, it has been proposed that preoperative CRT is a better treatment than postoperative CRT to enhance the rate

Received 10 June 2013, Revised 24 July 2013, Accepted 6 September 2013.

Correspondence: Hong Seok Jang, MD, Department of Radiation Oncology, Seoul St. Mary's Hospital, The Catholic University of Korea College of Medicine, 222 Banpo-daero, Seocho-gu, Seoul 137-701, Korea. Tel: +82-2-2258-1520, Fax: +82-2-2258-1532, E-mail: hsjang11@catholic.ac.kr

(c) This is an Open Access article distributed under the terms of the Creative Commons Attribution Non-Commercial License (http://creativecommons.org/ licenses/by-nc/3.0/) which permits unrestricted non-commercial use, distribution, and reproduction in any medium, provided the original work is properly cited.

www.e-roj.org 
of overall compliance, local control, and sphincter preservation and to reduce the risk of toxicity [5]. Due to these outcomes, preoperative CRT followed by radical surgery is widely regarded as the preferred treatment of choice for locally advanced rectal cancer.

When it comes to the assessment of rectal cancer, tumor responses, such as down-classification and pathologic complete response of primary tumors after preoperative CRT are known as prognostic factors [6-9]. Several studies have identified clinicopathological factors related to survival and recurrence following preoperative CRT [10-12]. Some trials have tried to verify survival benefit of preoperative CRT compared to postoperative CRT, but did not demonstrate significant improvements in disease-free survival (DFS) or OS $[4,5,13]$.

The aim of the present study was to evaluate the treatment outcomes of preoperative versus postoperative concurrent CRT on locally advanced rectal cancer.

\section{Materials and Methods}

\section{Patient characteristics}

One hundred fourteen patients with locally advanced rectal cancer who received preoperative CRT (54 patients) or postoperative CRT (60 patients) were analyzed retrospectively. All patients were treated at Seoul St. Mary's Hospital, the Catholic University of Korea from June 2003 through April 2011. Eligibility criteria included histologically confirmed rectal carcinoma, clinically or pathologically diagnosed stage II (T3 or T4 without any lymph node involvement) or stage III (any tumor stage with positive lymph node). Patients with a history of chemotherapy, radiotherapy, any other malignancies, or presence of distant metastasis at diagnosis were excluded.

The patient characteristics at baseline are shown in Table 1. There was no significant difference between the preoperative and postoperative CRT group with the exception of the distance of tumor from the anal verge. The ratio of patients whose tumor located close to the anal verge was higher in the preoperative CRT group than in the postoperative CRT group (57.4\% vs. 33.3\%, $p=0.01$ ). The median age was 59.5 years (range, 33 to 80 years). Clinical staging was classified by rectosigmoidoscopy, endosonography, computed tomography (CT) scan, and magnetic resonance imaging (MRI) of the abdomen and pelvis.

\section{Treatment}

5-Fluorouracil (5-FU) or a precursor of 5-FU-based chemo- therapy was conducted concurrently with radiotherapy for all patients. Most patients were treated using pelvic radiotherapy with the three- or four-field box techniques. Preoperative radiotherapy was delivered with a median total dose of 50.4 Gy (range, 45 to $55.8 \mathrm{~Gy}$ ) in a median of 28 fractions (range, 24 to 31 fractions). Meanwhile, postoperative radiotherapy was delivered with a median total dose of 50.4 Gy (range, 41.4 to $60.4 \mathrm{~Gy}$ ) in a median of 28 fractions (range, 23 to 33 fractions). The median duration of radiotherapy was 37 days (range, 21 to 56 days). All patients underwent total mesorectal excision. Surgical resection was performed at median 8 weeks (range, 5 to 12 weeks) after completion of radiotherapy in the preoperative CRT group. All patients of the preoperative CRT group received three or four cycles of adjuvant chemotherapy with the same preoperative regimen after the surgical resection. Postoperative radiotherapy was delivered at median 9 weeks (range, 1 to 14 weeks) following the surgical resection. Most (81.7\%) of the postoperative CRT group were administered one or two cycles of 5-FU or a precursor of 5-FU-based adjuvant chemotherapy before CRT. No patient had a relapse of rectal cancer at the start time of postoperative CRT. A precursor of 5-FU-based maintenance chemotherapy

Table 1. Patient characteristics $(n=114)$

\begin{tabular}{|c|c|c|c|}
\hline \multirow[b]{2}{*}{ Characteristic } & \multicolumn{2}{|c|}{ CRT arm } & \multirow[b]{2}{*}{ p-value } \\
\hline & $\begin{array}{l}\text { Preoperative } \\
\quad(n=54)\end{array}$ & $\begin{array}{l}\text { Postoperative } \\
\quad(n=60)\end{array}$ & \\
\hline Age (yr) & & & 0.10 \\
\hline $0-50$ & $9(16.7)$ & $18(30.0)$ & \\
\hline$>50$ & 45 (83.3) & $42(70.0)$ & \\
\hline Gender & & & 0.15 \\
\hline Male & $36(66.7)$ & 32 (53.3) & \\
\hline Female & $18(33.3)$ & $28(46.7)$ & \\
\hline Clinical tumor stage & & & 0.47 \\
\hline cT1 & $1(1.9)$ & $0(0)$ & \\
\hline cT2 & $5(9.2)$ & $9(15.0)$ & \\
\hline cT3 & $35(64.8)$ & $41(68.3)$ & \\
\hline cT4 & $13(24.1)$ & $10(16.7)$ & \\
\hline Clinical nodal stage & & & 0.72 \\
\hline Negative & $6(11.1)$ & 8 (13.3) & \\
\hline Positive & $48(88.9)$ & 52 (86.7) & \\
\hline $\begin{array}{l}\text { Distance of tumor } \\
\text { from anal verge }(\mathrm{cm})\end{array}$ & & & 0.01 \\
\hline $0-5$ & $31(57.4)$ & $20(33.3)$ & \\
\hline$>5$ & $23(42.6)$ & $40(66.7)$ & \\
\hline
\end{tabular}

Values are presented as number (\%). CRT, chemoradiotherapy. 


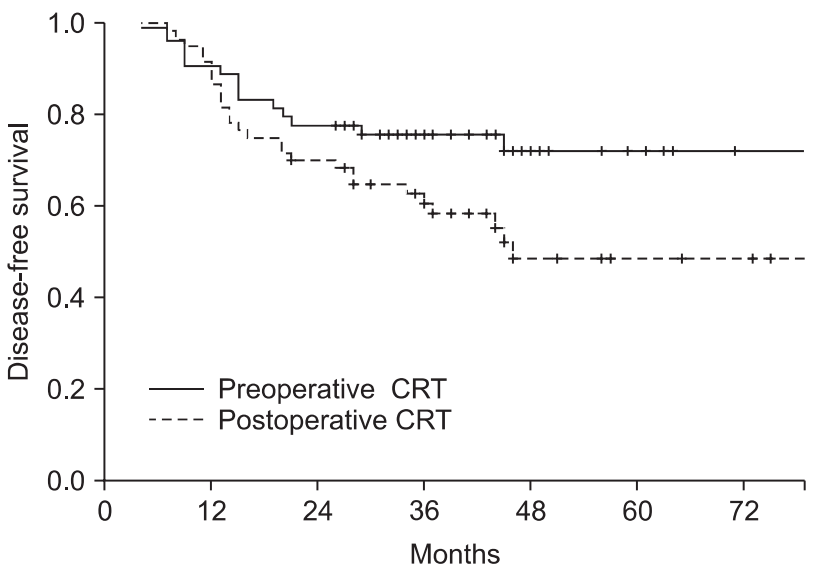

Fig. 1. Disease-free survival (DFS) of 114 patients who received preoperative or postoperative chemoradiotherapy (CRT). The DFS at five years was significantly higher in the preoperative CRT arm than in the postoperative CRT arm (72.1\% vs. 48.6\%; $p=0.05$ in the univariate analysis, $p=0.10$ in the multivariate analysis).

per oral was administered to $52.0 \%$ of the preoperative CRT group and $68.3 \%$ of the postoperative CRT group following completion of the adjuvant chemotherapy $(p=0.07)$.

\section{Follow-up and statistical analysis}

The follow-up duration was defined as the time from radiotherapy initiation in the preoperative CRT group or the day of surgery in the postoperative CRT group to last confirmation or death. The median follow-up duration was 44 months (range, 19 to 118 months) and 41 months (range, 16 to 100 months) for preoperative and postoperative CRT group, respectively $(p=0.31)$. Pathologic stage was classified using the TNM system. Follow-up evaluations included physical examination, complete blood count, biochemical profile, abdominopelvic CT (or MRI), proctoscopy, and chest radiography. Treatment-related toxicity was assessed by the criteria of the Radiation Therapy Oncology Group and the European Organization for Research and Treatment of Cancer. The criteria included the presence of proctitis, hematologic, dermatologic, and genitourinary effects for acute toxic effect and the presence of fistula, small bowel obstruction and pelvic abscess for chronic toxic effect. Grade 3 or higher toxic effects were considered meaningful. The primary end point was DFS. The secondary end points were OS, locoregional control, toxicity, and sphincter preservation rate. Each end point was measured from the time of treatment initiation. All survival rates were assessed and compared by using the KaplanMeier analysis and a log-rank test. Cox proportional hazards

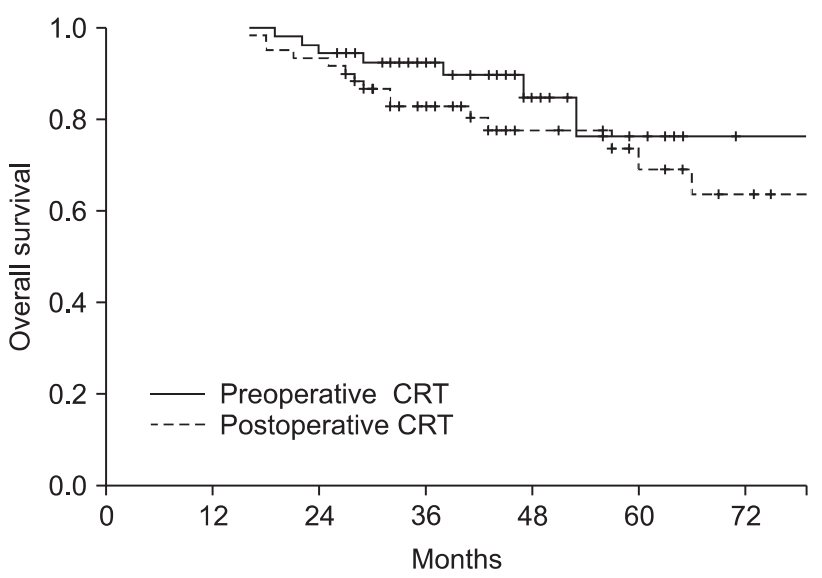

Fig. 2. Overall survival (OS) of 114 patients who received preoperative or postoperative chemoradiotherapy (CRT). The OS at five years was not significantly different between preoperative and postoperative arm (76.2 \% vs. $69.0 \% ; p=0.23$ ).

model was performed for multivariate analysis. For statistical analyses, SAS ver. 9.1 (SAS Institute, Cary, NC, USA) was used. A $p$-value $\leq 0.05$ was considered statistically significant.

\section{Results}

\section{DFS, OS and locoregional control rate}

The 5-year DFS rates was $72.1 \%$ for the preoperative CRT group, and $48.6 \%$ for the postoperative CRT group ( $p=0.05$, the univariate analysis; $p=0.10$, the multivariate analysis) (Fig. 1). The 5 -year OS rate was not significantly different in the group comparison (76.2\% vs. 69.0\%, p = 0.23) (Fig. 2). The 5 -year locoregional control rate was $85.2 \%$ for the preoperative CRT group and $84.7 \%$ for the postoperative CRT group ( $p=0.98$ ).

In the univariate analysis for 5-year DFS, preoperative CRT, pathologic node stage, low histologic grade tumor were statistically significant prognostic factors (Table 2). In the multivariate analysis, histologic grade was associated with DFS (Table 2).

\section{Sphincter preservation}

Among 51 patients with low-lying tumors (distance of tumor from the anal verge $\leq 5 \mathrm{~cm}$ ) which were considered to require an abdominoperineal resection, the sphincter preserving surgery was conducted $58.1 \%$ (18 of 31 ) in the preoperative CRT group patients, whereas $25.0 \%$ (5 of 20) of the postoperative patients $(p=0.02)$. The sphincter preservation probability between both groups is shown in Table 3 . 
Table 2. Prognostic factors for disease-free survival

\begin{tabular}{|c|c|c|c|c|}
\hline \multirow{2}{*}{ Variable } & \multirow{2}{*}{ No. of patient (\%) } & \multicolumn{3}{|c|}{ Disease-free survival } \\
\hline & & $5-y r(\%)$ & $p$-value & $p$-value $e^{b)}$ \\
\hline Age (yr) & & & 0.27 & - \\
\hline $0-50$ & $27(23.7)$ & 50.3 & & \\
\hline$>50$ & $87(76.3)$ & 62.2 & & \\
\hline Gender & & & 0.89 & - \\
\hline Male & $68(59.6)$ & 57.5 & & \\
\hline Female & $46(40.4)$ & 64.0 & & \\
\hline Modality & & & 0.05 & - \\
\hline Preoperative CRT & $54(47.4)$ & 72.1 & & \\
\hline Postoperative CRT & $60(52.6)$ & 48.6 & & \\
\hline Pathologic tumor stage & & & 0.14 & - \\
\hline yp or pT0-2 & $26(22.8)$ & 72.7 & & \\
\hline yp or pT3-4 & $88(77.2)$ & 55.7 & & \\
\hline Pathologic node stage & & & $<0.01$ & - \\
\hline Negative & $49(43.0)$ & 83.9 & & \\
\hline Positive & $65(57.0)$ & 41.0 & & \\
\hline Tumor location from anal verge $(\mathrm{cm})$ & & & 0.97 & - \\
\hline $0-5$ & $51(44.7)$ & 59.8 & & \\
\hline$>5$ & 63 (55.3) & 58.7 & & \\
\hline Histologic grade & & & $<0.01$ & $<0.01$ \\
\hline Low & 99 (86.8) & 63.8 & & \\
\hline High & $14(12.3)$ & 34.3 & & \\
\hline Unknown & $1(0.9)$ & - & & \\
\hline
\end{tabular}

CRT, chemoradiotherapy.

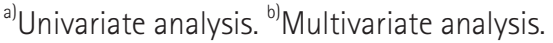

Table 3. Sphincter preservation

\begin{tabular}{lccc}
\hline Variable & Preoperative CRT $(n=54)$ & Postoperative CRT $(n=60)$ & $p$-value \\
\hline $\begin{array}{l}\text { Abdominoperineal resection considered necessary } \\
\text { (distance of tumor from anal verge } \leq 5 \mathrm{~cm})\end{array}$ & $31(57.4)$ & $20(33.3)$ & \\
$\begin{array}{l}\text { Sphincter preserving surgery performed } \\
\text { Values }\end{array}$ & $18 / 31(58.1)$ & $5 / 20(25.0)$ & 0.02 \\
\hline
\end{tabular}

Values are presented as number or (\%).

CRT, chemoradiotherapy.

\section{Pathologic characteristics}

The ypT status of preoperative CRT group consisted of ypTO (9 patients, 16.7\%), ypTis (2 patients, 3.7\%), ypT1 to ypT2 (9 patients, 16.7\%), ypT3 (33 patients, 61.1\%), and ypT4 (1 patients, 1.8\%). The ypN status of preoperative CRT group was ypNo (44 patients, 81.5\%), ypN1 (5 patients, 9.25\%), and ypN2 (5 patients, 9.25\%). Pathologic tumor and nodal downclassification were found after the preoperative CRT (53.7\% and $77.8 \%$, both $p<0.001)$. In the present study, pathologic complete response (CR) was defined as complete eradicated state of all viable tumor cells (i.e., pTONO). Nine patients (16.7\%) of the preoperative CRT group achieved pathologic CR. Sixteen patients (26.7\%) of the postoperative CRT group had the same tumor and nodal classifications before and after surgery. The pathologic characteristics are presented in Table 4.

\section{Toxicity}

The grade 3 or higher acute and chronic toxicities are displayed in Table 5. For both groups, most acute and chronic toxicities were mild to moderate. Grade 3 or higher acute toxicity was not significantly different between the two groups ( $p=$ 0.10). Three patients $(5.56 \%)$ in the preoperative CRT group 
Table 4. Pathologic tumor and nodal stage in both treatment groups

\begin{tabular}{cccc}
\hline & $\begin{array}{c}\text { Preoperative CRT } \\
(n=54)\end{array}$ & $\begin{array}{c}\text { Postoperative CRT } \\
(n=60)\end{array}$ & p-value \\
\hline $\begin{array}{c}\text { Tumor stage } \\
\text { pT0 }\end{array}$ & $9(16.7)$ & $0(0)$ & $<0.001$ \\
pTis & $2(3.7)$ & $0(0)$ & \\
pT1 & $2(3.7)$ & $2(3.3)$ & \\
pT2 & $7(13.0)$ & $4(6.7)$ & \\
pT3 & $33(61.1)$ & $43(71.7)$ & \\
pT4 & $1(1.8)$ & $11(18.3)$ & \\
Nodal stage & & & $<0.001$ \\
pN0 & $44(81.5)$ & $5(8.3)$ & \\
pN1 & $5(9.25)$ & $23(38.3)$ & \\
pN2 & $5(9.25)$ & $32(53.3)$ & \\
\hline
\end{tabular}

Values are presented as number (\%).

CRT, chemoradiotherapy.

had acute grade 3 or higher toxicity. Of the three patients, one had leukocytopenia, one had proctitis, and one had dermatitis. Nine patients (15.0\%) in the postoperative CRT group developed grade 3 or 4 acute toxicities; five patients had leukocytopenia, one patients had proctitis, one patient had nausea and vomiting, one patient had dermatitis, and one patient had genitourinary toxicity. All grade 3 or higher acute toxicities of both groups were reported during CRT. There was no significant variation of chronic toxicities between both groups (14.8\% vs. $10.0 \%, p=0.62$ ).

\section{Discussion and Conclusion}

Many studies have reported the efficacy of preoperative CRT for the patients with locally advanced rectal cancer $[4,5,10,13-$ 17]. Preoperative CRT results in higher rate of sphincter preservation than does postoperative CRT $[5,13]$. The rate of sphincter-preserving surgery after preoperative CRT varies widely $[5,16]$. Among the patients who were considered to require an abdominoperineal resection in preoperative CRT group of German rectal cancer trial, the rate of sphincter preservation was 39\% [5]. However, we observed a sphincter preservation rate of $58.1 \%$ in the preoperative CRT group. The results of the present study correspond well with the findings of earlier experimental studies that documented high rate of tumor regression. Accordingly, tumor size decrease leads to sphincter preservation and tumor respectability $[14,15]$.

Tumor response after preoperative CRT is known as a prognostic factor in rectal cancer [6-9]. The level of response
Table 5. Grade 3 or higher acute and chronic toxicity

\begin{tabular}{lccc}
\hline & $\begin{array}{c}\text { Preoperative } \\
\text { CRT }(n=54)\end{array}$ & $\begin{array}{c}\text { Postoperative } \\
\text { CRT }(n=60)\end{array}$ & p-value \\
\hline Acute & $3(5.56)$ & $9(15.0)$ & 0.10 \\
$\quad$ Proctitis & $1(1.85)$ & $1(1.7)$ & 0.94 \\
Hematologic effects & $1(1.85)$ & $5(8.3)$ & 0.12 \\
Dermatologic effects & $1(1.85)$ & $1(1.7)$ & 0.94 \\
Genitourinary effects & $0(0)$ & $1(1.7)$ & 0.34 \\
Nausea/vomiting & $0(0)$ & $1(1.7)$ & 0.34 \\
Chronic & $7(14.8)^{*}$ & $6(10.0)^{+}$ & 0.62 \\
Fistula & $2(3.7)$ & $2(3.3)$ & 0.92 \\
Small bowel obstruction & $3(5.55)$ & $4(6.7)$ & 0.81 \\
Pelvic abscess & $3(5.55)$ & $3(5.0)$ & 0.89 \\
\hline
\end{tabular}

Values are presented as number (\%).

CRT, chemoradiotherapy.

*In the preoperative CRT group, one patient had small bowel obstruction and fistula. ${ }^{\dagger}$ In the postoperative CRT group, one patient had pelvic abscess and fistula, and other two patients had pelvic abscess and small bowel obstruction.

(CR vs. partial response vs. no response) significantly influences local recurrence, DFS, freedom from distant metastases, and cancer specific survival $[10,17]$. Park et al. [18] suggested that the patients who had good response (ypTO-2, N0) to preoperative CRT have a lower risk than poor responders in local and distant failure. Nine patients (16.7\%) of the preoperative CRT group achieved pathologic $C R$, and 18.5\% displayed lymph node involvement in the current study.

Preoperative CRT in the German trial could not bring significant advantages to DFS or OS: Five-year OS was $76 \%$ vs. $74 \%(p=0.80)$ and 5 -year DFS rate was 68\% vs. 65\% ( $p=0.32)$ in the preoperative and postoperative CRT group, respectively [5]. In the National Surgical Adjuvant Breast and Bowel Project (NSABP) R-03 trial, 5-year OS was 74.5\% vs. 65.6\% ( $p=$ $0.065)$ and 5 -year DFS was $64.7 \%$ vs. $53.4 \%(p=0.011)$ in the preoperative and postoperative CRT group, respectively [4]. Roh et al. [4] attributed the prolonged DFS of NSABP R-03 to patient eligibility, timing of radiotherapy, radiation dose, type of surgery, and the length of follow-up. In the current study, the 5-year DFS rate was $72.1 \%$ and $48.6 \%$ for the preoperative and postoperative CRT group, respectively ( $p=0.05$, the univariate analysis; $p=0.04$, the multivariate analysis), and the 5 -year OS was not significantly different between both groups (76.2\% vs. 69.0\%, $p=0.23$ ). The 5 -year locoregional control rate was $85.2 \%$ for the preoperative CRT group and $84.7 \%$ for the postoperative CRT group ( $p=0.98$ ). It is likely that the results of survival and local control can be explained 
by the difference of cancer stage distribution and relatively small number of patients compared to previous studies. There may be a propensity for selection bias of including more patients with poor prognosis in the postoperative CRT group than the preoperative CRT group. The Action to Control Cardiovascular Risk in Diabetes (ACCORD) 12 trial attempted to improve the complete remission rate with the intensified chemotherapy regimens. The study achieved the complete remission rate up to $19.2 \%$ and the remission rate with few residual cells up to 40\% after CRT [19]. Nonetheless, there was no significant improvement in survival [20]. The proportional difference of maintenance chemotherapy between both groups was not statistically significant ( $p=0.07)$, so maintenance chemotherapy was not likely to affect the outcome. Median one cycle (56.7\%; range, 0 to 2 cycles) of 5 -FU or a precursor of 5-FU-based adjuvant chemotherapy was administered before postoperative CRT in our institution. In the early 2000s, first two cycles of chemotherapy were administered before postoperative CRT according to the North Central Cancer Treatment Group study [3,21,22], but early postoperative CRT has gradually increased in our institution since Lee et al. [23] demonstrated that early postoperative CRT has a significant advantage for DFS compared with late postoperative CRT.

Although gastrointestinal toxicity was not appreciably different between both groups, preoperative CRT reduces radiation dose to the small bowel. Thus, it reduces risk of radiation enteritis [24]. We could not find any statistically significant differences between grade 3 or higher acute and chronic toxicities of both groups $(p=0.10$ and $p=0.62$, respectively). Possible reason for insignificantly different toxicities between both groups may be associated with small number of enrolled patients, high proportion (95\%) of patients who received planned postoperative CRT and surgeon-related variation. All grade 3 or higher acute toxicities of both groups were reported during CRT in the current study, reflecting that concurrent CRT increases toxicity [25].

Collectively, although there were no advantages of DFS, OS, locoregional control rate, acute and chronic toxicities in the preoperative CRT, preoperative CRT significantly improved the down-classification rate and the sphincter preservation of low-lying tumors in rectal cancer.

\section{Conflict of Interest}

No potential conflict of interest relevant to this article was reported.

\section{Acknowledgments}

This work was supported by a grant from the National RetD Program for Cancer Control, Ministry of Health \& Welfare, Republic of Korea (0820010).

\section{References}

1. Jung KW, Park S, Kong HJ, et al. Cancer statistics in Korea: incidence, mortality, survival, and prevalence in 2009. Cancer Res Treat 2012;44:11-24.

2. Gastrointestinal Tumor Study Group. Prolongation of the disease-free interval in surgically treated rectal carcinoma. N Engl J Med 1985;312:1465-72.

3. Krook JE, Moertel CG, Gunderson LL, et al. Effective surgical adjuvant therapy for high-risk rectal carcinoma. N Engl J Med 1991;324:709-15.

4. Roh MS, Colangelo LH, O'Connell MJ, et al. Preoperative multimodality therapy improves disease-free survival in patients with carcinoma of the rectum: NSABP R-03. J Clin Onco 2009;27:5124-30.

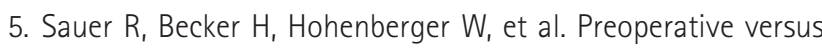
postoperative chemoradiotherapy for rectal cancer. N Engl J Med 2004;351:1731-40.

6. Ruo L, Tickoo S, Klimstra DS, et al. Long-term prognostic significance of extent of rectal cancer response to preoperative radiation and chemotherapy. Ann Surg 2002;236:75-81.

7. Capirci C, Valentini V, Cionini L, et al. Prognostic value of pathologic complete response after neoadjuvant therapy in locally advanced rectal cancer: long-term analysis of 566 ypCR patients. Int J Radiat Oncol Biol Phys 2008;72:99-107.

8. Rosenberg R, Nekarda H, Zimmermann F, et al. Histopathological response after preoperative radiochemotherapy in rectal carcinoma is associated with improved overall survival. J Surg Oncol 2008;97:8-13.

9. Rullier A, Laurent C, Capdepont M, et al. Impact of tumor response on survival after radiochemotherapy in locally advanced rectal carcinoma. Am J Surg Pathol 2010;34:562-8.

10. Rodel C, Martus P, Papadoupolos T, et al. Prognostic significance of tumor regression after preoperative chemoradiotherapy for rectal cancer. J Clin Oncol 2005;23:8688-96.

11. Song JH, Jang HS, Kim YS, et al. The pathological and clinical effects of preoperative chemoradiation in rectal cancer. $J$ Korean Soc Ther Radiol Oncol 2011;29:11-9.

12. Yoon MS, Nam TK, Kim HR, et al. Results of preoperative concurrent chemoradiotherapy for the treatment of rectal cancer. J Korean Soc Ther Radiol Oncol 2008;26:247-56.

13. Park JH, Yoon SM, Yu CS, et al. Randomized phase 3 trial comparing preoperative and postoperative chemoradiotherapy 
with capecitabine for locally advanced rectal cancer. Cancer 2011;117:3703-12.

14. Chen ET, Mohiuddin M, Brodovsky H, Fishbein G, Marks G. Downstaging of advanced rectal cancer following combined preoperative chemotherapy and high dose radiation. Int J Radiat Oncol Biol Phys 1994;30:169-75.

15. Janjan NA, Khoo VS, Abbruzzese J, et al. Tumor downstaging and sphincter preservation with preoperative chemoradiation in locally advanced rectal cancer: The M. D. Anderson Cancer Center experience. Int J Radiat Oncol Biol Phys 1999;44:102738.

16. Lee JH, Kim SH, Kim JG, et al. Preoperative chemoradiotherapy (CRT) followed by laparoscopic surgery for rectal cancer: predictors of the tumor response and the long-term oncologic outcomes. Int J Radiat Oncol Biol Phys 2011;81:431-8.

17. Janjan NA, Crane C, Feig BW, et al. Improved overall survival among responders to preoperative chemoradiation for locally advanced rectal cancer. Am J Clin Oncol 2001;24:107-12.

18. Park IJ, You YN, Skibber JM, et al. Comparative analysis of lymph node metastases in patients with ypTo-2 rectal cancers after neoadjuvant chemoradiotherapy. Dis Colon Rectum 2013;56:135-41.

19. Gerard JP, Azria D, Gourgou-Bourgade $S$, et al. Comparison of two neoadjuvant chemoradiotherapy regimens for locally advanced rectal cancer: results of the phase III trial ACCORD 12/0405-Prodige 2. J Clin Oncol 2010;28:1638-44.

20. Gerard JP, Azria D, Gourgou-Bourgade S, et al. Clinical outcome of the ACCORD 12/0405 Prodige 2 randomized trial in rectal cancer. J Clin Oncol 2012;30:4558-65.

21. Douglass HO Jr, Moertel CG, Mayer RJ, et al. Survival after postoperative combination treatment of rectal cancer. $\mathrm{N}$ Engl J Med 1986;315:1294-5.

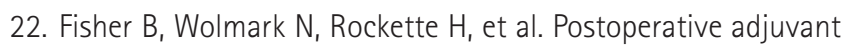
chemotherapy or radiation therapy for rectal cancer: result from NSABP R-01. J Natl Cancer Inst 1988;80:21-9.

23. Lee JH, Lee JH, Ahn JH, et al. Randomized trial of postoperative adjuvant therapy in stage II and III rectal cancer to define the optimal sequence of chemotherapy and radiotherapy: a preliminary report. J Clin Oncol 2002;20:1751-8.

24. Minsky BD, Conti JA, Huang Y, Knopf K. Relationship of acute gastrointestinal toxicity and the volume of irradiated small bowel in patients receiving combined modality therapy for rectal cancer. J Clin Oncol 1995;13:1409-16.

25. Choy $H$, MacRae R, Story M. Basic concepts of chemotherapy and irradiation interaction. In: Halperin EC, Perez CA, Brady LW, editors. Perez and Brady's principles and practice of radiation oncology. 5th ed. Philadelphia: Lippincott Williams \& Wilkins; 2008. p. 672-73. 